MR. JUAN IGNACIO ELIO MARIÁNGELO (Orcid ID : 0000-0002-5757-2531)

Article type : Regular Paper

\title{
Chemical chaperones improve the functional recovery of stunned myocardium by attenuating the endoplasmic reticulum stress
}

\begin{abstract}
Mariángelo, Juan Ignacio Elio; Román, Bárbara; Silvestri, María Agustina; Salas, Margarita; Vittone, Leticia; Said, Matilde; Mundiña-Weilenmann, Cecilia.
\end{abstract}

Centro de Investigaciones Cardiovasculares, CCT-CONICET La Plata, Facultad de Ciencias Médicas, Universidad Nacional de La Plata, Argentina.

Short title: UPR in stunned myocardium

Corresponding author: Dr. Cecilia Mundiña-Weilenmann

Centro de Investigaciones Cardiovasculares

Facultad de Ciencias Médicas

60 y 120, 1900 La Plata, Argentina.

Tel/Fax: 54-221-4834833

Email: cmundweil@med.unlp.edu.ar

\section{ABSTRACT}

Aim

Myocardial ischemia/reperfusion (I/R) produces structural and functional alterations depending on the duration of ischemia. Brief ischemia followed by reperfusion causes reversible contractile dysfunction (stunned heart) but long-lasting ischemia followed by reperfusion can result in irreversible injury with cell death. Events during I/R can alter endoplasmic reticulum (ER) function leading to the accumulation of unfolded/misfolded proteins. The resulting ER stress induces activation of several signal transduction pathways, known as unfolded protein response (UPR). Experimental evidence shows that UPR contributes to cell death in irreversible I/R injury, however there is still uncertainty for its occurrence in the stunned myocardium. This study investigated the ER

This article has been accepted for publication and undergone full peer review but has not been through the copyediting, typesetting, pagination and proofreading process, which may lead to differences between this version and the Version of Record. Please cite this article as doi: 10.1111/apha.13358

This article is protected by copyright. All rights reserved. 
stress response and its functional impact on the post-ischemic cardiac performance of the stunned heart.

\section{Methods}

Perfused rat hearts were subjected to $20 \mathrm{~min}$ of ischemia followed by $30 \mathrm{~min}$ of reperfusion. UPR markers were evaluated by qRT-PCR and Western blot. Post-ischemic mechanical recovery was measured in absence and presence of two chemical chaperones: tauroursodeoxycholic acid (TUDCA) and 4-phenylbutyric acid (4-PBA).

\section{Results}

Analysis of mRNA and protein levels of various ER stress effectors demonstrated that different UPR signaling cascades, involving both pro-survival and pro-apoptotic pathways, are activated. Inhibition of the UPR with chemical chaperones improved the post-ischemic recovery of cardiac mechanical function without affecting the $\mathrm{l} / \mathrm{R}$-induced increase in oxidative stress.

\section{Conclusion}

Our results suggest that prevention of ER stress by chemical chaperones could be a therapeutic tool to limit deterioration of the contractile function in clinical settings in which the phenomenon of myocardial stunning is present.

\section{MYOCARDIAL ISCHEMIA/REPERFUSION - ENDOPLASMIC RETICULUM STRESS - CHEMICAL CHAPERONES}

\section{INTRODUCTION}

Restoration of coronary flow after an ischemic event is mandatory in order to preserve cardiac function. However reperfusion itself may result in mechanical, electrical and/or viability alterations in excess to that produced by ischemia alone. The resulting tissue damage is named ischemia/reperfusion (I/R) injury and depends mainly on the duration of the ischemia. If the ischemic period is brief, I/R can cause reversible contractile dysfunction and arrhythmias (stunned heart) while in its severest form (prolonged ischemia) I/R leads to cell death ${ }^{1}$.

The endoplasmic reticulum (ER) is the main organelle involved in the proper folding and sorting of newly synthesized secretory and membrane proteins. Events occurring during I/R such as depletion of oxygen/glucose supply, $\mathrm{Ca}^{2+}$ mishandling and reactive oxygen species (ROS) accumulation interfere with ER correct function leading to the accumulation of unfolded/misfolded proteins, a condition referred to as ER stress ${ }^{2,3}$. ER stress triggers the unfolded protein response (UPR) consisting in various transcriptional and translational pathways which orchestrate the restoration of ER homeostasis in three distinct ways: 1 ) by upregulating the transcription of chaperones; 2) by attenuating the translation and therefore protein accumulation into the ER and 3) by facilitating retrotranslocation of proteins from the ER to the citosol for ubiquitin-proteasome dependent or autophagy-lysosome dependent degradation ${ }^{2,4}$. However, during prolonged or overwhelming protein folding stress, the adaptation of UPR starts to fail and a switch to pro-apoptotic signals generates the death of irreversible damaged cells.

This article is protected by copyright. All rights reserved. 
The UPR signaling cascade relies on three independent but overlapping ER stress sensors: protein kinase RNA-like ER kinase (PERK), activating transcription factor 6 (ATF6) and inositol-requiring enzyme 1 (IRE1 $\alpha)^{2,5}$. Under non-stressed conditions, these proteins are inhibited by the binding of glucose-regulated protein of $78 \mathrm{kDa}$ (GRP78), an ER resident chaperone. Upon ER stress, the unfolded proteins recruit the chaperone away from the sensors leading to the activation of specific downstream pathways. PERK triggers the phosphorylation of the eukaryotic initiation factor 2 (elF2 $\alpha$ ), which inhibits global protein synthesis, except for some specific proteins such as Activating Transcription Factor 4 (ATF4) and GRP78. Prolonged or extreme ER stress uses ATF4 to increase CCAAT/-enhancer-binding protein homologous protein (CHOP) transcription, an apoptotic signaling molecule. ATF6 is activated by cleavage after being transported to the Golgi, and then translocates to the nucleus where it selectively increases the expression of chaperones and ER-associated degradation (ERAD) proteins, as well as X-box-binding protein 1 (XBP1). Finally, IRE1 $\alpha$ due to its endoribonuclease activity mediates the unconventional splicing of the XBP1 mRNA for its translation into an active transcription factor, the spliced XBP1 (sXBP1), which reinforces the gene expression of chaperones and ERAD proteins. In addition, IRE1 $\alpha$ stimulates the stress-activated c-Jun aminoterminal kinase (JNK) and activates the ER localized caspase-12, connecting ER stress with apoptosis and autophagy. Overall, the UPR controls cell survival as well as the execution of apoptosis. The finetuning of these processes will decide the ER stress-induced cell fate.

In the heart under severe ischemic insult, the activation of all three branches of the UPR has been reported ${ }^{6}$. The participation of ATF6 was demonstrated by a substantial amount of research using both pharmacologic and genetic approaches in in vitro and in vivo studies ${ }^{7-10}$. Overall the evidence supported a cardioprotective role of this pathway against I/R damage mediated not only by inducing genes that encode ER resident chaperones and components of the ERAD machinery ${ }^{11,12}$, but also through the ATF6-induced expression of SERCA2a and antioxidant genes, allowing a link between ER stress and oxidative stress and $\mathrm{Ca}^{2+}$ handling ${ }^{13,14}$. Regarding the PERK branch, Miyazaki et al. showed that $\mathrm{I} / \mathrm{R}$ activates the phosphorylation of elF $2 \alpha$ and increases CHOP gene expression ${ }^{3}$. Moreover, they demonstrated that hearts from $\mathrm{CHOP}$-deficient mice exhibited markedly reduced reperfusion injury when compared with wild-type mice, associated to a decreased number of apoptotic cardiomyocytes, suggesting a deleterious effect of CHOP-mediated pathway on the development of I/R injury. Finally, the specific role for the IRE1 $\alpha$ signaling was addressed in gain- and loss-of-function studies of its downstream component SXBP1. Wang et al. ${ }^{15}$ demonstrated that SXBP1 expression is necessary and sufficient to protect the heart from I/R injury. Although it is known that persistent stress can cause the activation of pro-apoptotic pathways of the IRE $1 \alpha$ cascade ${ }^{16,17}$, this has not yet been clarified in the context of myocardial I/R injury.

All these studies confirmed that UPR is triggered in the myocardium under those ischemic challenges that lead to cell death. However, there is still a gap for the occurrence of ER stress in an ischemic condition not associated with irreversible cell damage. This prompted us to investigate whether UPR is activated in the stunned heart and to examine the signaling pathways involved. In addition, we evaluated the functional impact of the ER stress response in the post-ischemic cardiac performance of the stunned heart.

This article is protected by copyright. All rights reserved. 


\section{RESULTS}

ER stress in the stunned heart

To ascertain whether UPR is triggered in the stunned heart, the relative abundance of mRNA encoding several ER stress markers (sXBP1, total-XBP1, GRP78 and CHOP) was analyzed by real-time quantitative RT-PCR (Figure 1). Compared with non-ischemic hearts, hearts subjected to I/R 20/30 showed a significant increase in SXBP1, total-XBP1and GRP78 mRNA levels. This enhancement was similar to that detected in hearts subjected to I/R 30/60. However, the levels of CHOP mRNA, which were significantly elevated in hearts with irreversible I/R damage, showed no increase in stunned hearts when compare with non-ischemic hearts. To test whether this lack of increase in CHOP mRNA levels was due to the reperfusion time point at which the analysis was performed, a group of hearts was subjected to a prolonged reperfusion period of $60 \mathrm{~min}$ following the $20 \mathrm{~min}$ of ischemia. Once again, the expression of CHOP mRNA levels remained unchanged with respect to non-ischemic hearts, while mRNA levels of the adaptive UPR genes were comparable to those obtained after $\mathrm{I} / \mathrm{R}$ 20/30 (Figure S1). These results suggest that is the intensity of the ischemic insult, and not the reperfusion time, what determines the outcome of the ER stress response. Taken together, the results at the level of mRNA indicate that the UPR is triggered in the stunned heart, with signaling pathways belonging to the adaptive cascades for cell survival. The main difference between the UPR induced by the two I/R protocols was that in hearts with severe I/R injury, the adaptive UPR signals were concurrently activated with the transcriptional induction of $\mathrm{CHOP}$, a key pro-apoptotic component of the ER stress response.

Since prolonged simulated ischemia alone has been shown to efficiently activate certain ER stress response genes in neonatal rat cardiac myocytes ${ }^{7,9}$, we investigated if $20 \mathrm{~min}$ of ischemia were sufficient to upregulate the expression of GRP78 and SXBP1 in the ex vivo perfused heart. As depicted in Figure S2, ischemia elicited an increase in GRP78 but not in SXBP1 mRNA levels. These results support the concept that UPR pathways have different temporal dynamics. Our findings do not allow us to conclude if more time of ischemia alone or conditions provided by reperfusion, are required for the induction of SXBP1.

We then studied proteins involved in the initial phase of ER stress response ${ }^{18}$ : the phosphorylation of elF $2 \alpha$, associated to the rapid halt in protein translation and the activation of JNK and caspase-12, components of the ER stress-mediated apoptotic pathway (Figures $2 \mathrm{~A}$ and $\mathrm{B}$ ). The phosphorylation of elF $2 \alpha$ increased in stunned hearts with respect to non-ischemic hearts but not in hearts with irreversible I/R damage. No changes were detected in total elF2 $\alpha$ (Figure S3A and $C$ ). The phosphorylation of JNK increased in both I/R groups, with values significant higher in the I/R 20/30 than in the I/R 30/60. Finally, caspase- 12 was similarly activated under mild and severe I/R injuries compare to the non-ischemic group.

The results obtained at the protein level in the stunned heart demonstrate the activation of an early marker of the adaptive UPR branch (elF2 $\alpha$ phosphorylation) and unexpectedly, the simultaneous activation of two proteins generally related to pro-apoptotic cascades of the UPR (JNK and caspase12).

This article is protected by copyright. All rights reserved. 


\section{$I / R$-induced injury}

To assess whether the activation of pro-apoptotic cascades was leading to cell death in hearts subjected to I/R 20/30, we performed TUNEL staining of left ventricular sections obtained at the end of the protocol. Figures $3 \mathrm{~A}$ and $\mathrm{B}$ showed that the number of myocytes undergoing apoptosis (TUNEL-positive nuclei) were not different from non-ischemic hearts, even when the reperfusion period was extended to $60 \mathrm{~min}$ following the $20 \mathrm{~min}$ ischemia. Meanwhile, TUNEL-positive nuclei significantly increased in hearts subjected to I/R 30/60. Permanent cardiac damage was examined by TTC staining. The results showed an infarct size of $58.2 \pm 6.3 \%$ in hearts subjected to the severe $I / R$ injury while the necrotic area was negligible in stunned and non-ischemic perfused hearts (Figure 4A). Additionally, cell cytotoxicity was evaluated by LDH release assay. As depicted in Figure 4B, the increase in LDH levels in the perfusate obtained at 10 min of reperfusion, attained statistical significance only in those hearts subjected to the protocol of irreversible I/R injury. These results were in agreement with the TTC staining findings and are consistent with the generally accepted understanding that in rats an ischemic period up to 20 min causes minimal tissue damage ${ }^{1}$.

In the absence of detectable apoptosis, the overall results suggest that the UPR maladaptive pathways triggered by the stunned heart are either ineffective or overwhelmed by the pro-survival pathways.

\section{Functional impact of UPR in the stunned myocardium}

To establish the functional impact of the ER stress on the post-ischemic recovery of the stunned myocardium, we used the chemical chaperone, TUDCA. We first tested the ability of TUDCA to alleviate the activation of the UPR in our experimental condition by measuring the expression of ER stress related genes in hearts subjected to $I / R 20 / 30$ in the presence of the chaperone. As shown in Figure 5A, TUDCA significantly prevented the increase in SXBP1 and GRP78 mRNA levels detected in $I / R$ untreated hearts, confirming the effectiveness of TUDCA as a UPR inhibitor. Figures 5B-D shows the time course of different mechanical parameters used to characterize left ventricular function during I/R. TUDCA treatment significantly improved the post-ischemic recovery of contractility, measured by the maximum rate of pressure development $(+\mathrm{dP} / \mathrm{dt})$ and accelerated relaxation, evidenced by the shortening of half-relaxation time $\left(t_{1 / 2}\right)$, compared with the untreated hearts (Figures 5B and C). In addition, TUDCA mitigated the reperfusion-induced contracture, as indicated by the reduction of left ventricular end diastolic pressure (LVEDP, Figure 5D). To confirm the cardioprotective role of ER stress inhibition in the stunned heart, we studied the effects of another chemical chaperone, 4-PBA. As shown in Figures 6A-D, the prevention of $\mathrm{I} / \mathrm{R}$-induced enhancement of UPR markers by 4-PBA occurred in association with increased contractile performance, accelerated relaxation and decreased reperfusion-induced contracture. Neither TUDCA nor 4-PBA affected basal mechanical function (Table 1 in Supporting Information).

Since TUDCA and 4-PBA have been reported to show cytoprotective actions by reducing the oxidative stress ${ }^{19,20}$, we evaluated their effects on the I/R-induced oxidative stress. Lipid peroxidation products (thiobarbituric acid reactive substances, TBARS) were measured as a marker of oxidative injury. As shown in Figure 6E, the amount of TBARS significantly increased after I/R in the untreated group with respect to non-ischemic hearts, and this was unaltered by TUDCA or 4-PBA administration. Overall, the results indicate that inhibition of the UPR can improve the post-ischemic recovery of cardiac mechanical function. Moreover, the protection exerted by TUDCA and 4-PBA against I/R-associated mechanical dysfunction seems to be independent of the oxidative stress.

This article is protected by copyright. All rights reserved. 


\section{DISCUSSION}

Brief periods of myocardial ischemia not causing irreversible damage may nevertheless result in a transient impairment of contractile function and in the appearance of lethal arrhythmias upon reperfusion. The molecular mechanisms that have been proposed to underlie this potentially serious deterioration of myocardial performance include reactive oxygen species (ROS) generation and intracellular $\mathrm{Ca}^{2+}$ overload ${ }^{21}$. These two cellular perturbations are also known to interfere with ER proper function triggering the UPR ${ }^{2,22}$. In the context of severe $I / R$ injury, evidence has been presented that ER stress can either mitigate or cause myocardial damage ${ }^{6}$. However, there have been no studies examining the ER stress response and the cellular processes regulated by ER stress in the stunned myocardium. The data presented here demonstrated that an ischemic insult of 20 min in perfused rat hearts is sufficient to trigger the UPR. Analysis of mRNA and protein levels of different ER stress effectors allowed us to suggest that all 3 branches of the UPR are activated in the stunned heart: increased phosphorylation of elF $2 \alpha$ is a molecular readout of PERK activation; overexpression of unspliced XBP1 is a specific marker of ATF6 activity and elevated SXBP1, the consequence of IRE1 $\alpha$ endoribonuclease activity. Furthermore, IRE1 $\alpha$ pathway could also be involved in elevation of both, JNK phosphorylation and cleavage of caspase-12. Finally, increased GRP78 mRNA level, a well characterized biomarker of ER stress, could not be attributed to a specific UPR branch since it is a target gene of all the three axes. Most of these UPR downstream signals are involved in events that target cellular protective responses ${ }^{6}$. However, at first sight it seems contradictory that caspase-12 and JNK, two molecules initially linked to ER-induced apoptosis ${ }^{17,23,24}$ appeared activated in an I/R injury with minimal or no myocardial damage. Different experiments using cells lacking caspase-12 or in caspase-12 deficient mice demonstrated that the ER-resident caspase does not actively contribute to apoptosis ${ }^{25-28}$. Caspase-12 appears to have very limited enzymatic activity and specifically directed towards its own proenzyme and not to any other caspase of the apoptotic cascade ${ }^{29}$. Thus, caspase- 12 processing may constitute just an epiphenomenon of the UPR. Nevertheless, it is still often used as a marker for ER-stress. The role of JNK activation in the ER stress response has also been reconsidered ${ }^{30-32}$. JNK signaling is currently accepted to have functionally distinct phases with opposing effects in the ER stress response. In contrast to the proapoptotic late phase, the early and transient phase of JNK activation has been reported to protect ER-stressed cells from executing apoptosis ${ }^{30,33}$. The mechanisms involved in this pro-survival signaling response include the JNK-induced expression of antiapoptotic genes ${ }^{30}$ and the JNKmediated induction of autophagic flux ${ }^{31,32}$. Taken together, these reported results allow us to speculate that under the sub-cytotoxic ER stress induced by the stunned heart both, caspase-12 activation and JNK-phosphorylation, are either not playing any role in apoptosis or leading to protective cascades during the ER stress response.

The involvement of the ER stress response in different pathologies has been inferred from studies using animals with genetic manipulation of UPR central genes or by the use of pharmacological tools such as the chemical chaperones, 4-phenylbutyric acid (4-PBA) and tauroursodeoxycholic acid (TUDCA) ${ }^{34,35}$. In perfused rat hearts submitted to a severe I/R injury (ischemic period of 30 min or longer), the inhibition of the UPR by 4-PBA or TUDCA, decreased the number of apoptotic myocytes and diminished the infarct size, alleviating the deterioration of cardiac contractile function ${ }^{19,36-39}$. In some cases, these beneficial effects were associated to a reduction in the oxidative stress ${ }^{19}$. The present results show for the first time that during a mild I/R injury, with minimal or no cell death, the

This article is protected by copyright. All rights reserved. 
inhibition of the ER stress response is also cardioprotective, suggesting that the UPR is contributing to the decline of the post-ischemic mechanical performance of the stunned heart. Since the TUDCA and 4-PBA-induced contractile improvement occurred despite not having any effect on the I/Rinduced oxidative stress, it is tempting to speculate that UPR inhibitors are ameliorating the intracellular $\mathrm{Ca}^{2+}$ mishandling occurring upon reperfusion.

Previous studies from our group performed in Langendorff-perfused mouse hearts subjected to an I/R stunned protocol showed a sarcoplasmic reticulum (SR) $\mathrm{Ca}^{2+}$ depletion coupled to a decreased $\mathrm{Ca}^{2+}$ transient occurring upon reperfusion ${ }^{40}$. The findings suggest that this $\mathrm{SR} \mathrm{Ca}^{2+}$ leak is responsible at least in part for the cytosolic $\mathrm{Ca}^{2+}$ overload at the onset of reflow and thus, to the contractile dysfunction described for the stunned heart. Moreover, thapsigargin or tunicamycin, two wellknown ER stressors, have been reported to induce the release of $\mathrm{Ca}^{2+}$ from the ER in different cell lines ${ }^{41-43}$. Therefore, it is interesting to speculate that the I/R-induced ER stress is contributing to the $\mathrm{SR} / \mathrm{ER} \mathrm{Ca}^{2+}$ loss and in consequence to the intracellular $\mathrm{Ca}^{2+}$ alterations and contractile impairment upon reperfusion. At this respect, it has been suggested that the translocon, one of the $\mathrm{ER} \mathrm{Ca}^{2+}$ leak mechanisms ${ }^{44}$, mediates the ER Ca ${ }^{2+}$ unloading that occurred in response to ER stress inducers ${ }^{41-43}$. The translocon is a protein complex involved in protein transport that under non-stress conditions is sealed from the luminal side by GRP78 but when the chaperone is recruited by the unfolded proteins, it behaves as a $\mathrm{Ca}^{2+}$ channel ${ }^{41,43,45}$. Whether the translocon is present in cardiac muscle and whether it plays a functional role during the I/R-induced ER stress, remains to be determined.

In conclusion, our results showed that ER stress is triggered in the stunned heart and contributes to the contractile dysfunction. Furthermore, our data suggest that prevention of the ER stress by the use of chemical chaperones could be a therapeutic tool to limit the deterioration of the contractile function in clinical settings such as spontaneous reperfusion after revascularization therapies or after thrombolysis subsequent to acute myocardial infarction ${ }^{46}$.

\section{Materials and Methods}

\section{Animals}

The experiments were performed in male Wistar rats (200-300 g body weight). Animals were inbreeded and maintained in our animal facilities in accordance with the Guide for the Care and Use of Laboratory Animals (NIH, 2011). The protocols were approved by the Institutional Animal Care and Use Committee (CICUAL) of School of Medicine, National University of La Plata, Argentina (Nro T05022014).

\section{Ex vivo experiments: intact hearts}

During this study, animals were anaesthetized by intraperitoneal injection of Ketamine/Xylazine $(75 \mathrm{mg} / \mathrm{kg} / 10 \mathrm{mg} / \mathrm{kg})$. The dose was sufficient to produce a surgical level of anesthesia (loss of pedal withdrawal reflex) without profoundly affecting the cardiovascular function. Central thoracotomy and heart excision were performed immediately after phase III of anesthesia was reached. Isolated hearts were perfused at constant temperature $\left(37^{\circ} \mathrm{C}\right)$, heart rate $(4 \mathrm{~Hz})$ and flow $(14 \mathrm{ml} / \mathrm{min})$ with physiological bicarbonate buffer solution (BBS) composed by (in $\mathrm{mM}$ ): $128.3 \mathrm{NaCl}, 4.7 \mathrm{KCl}, 1.35$ $\mathrm{CaCl}_{2}, 20.2 \mathrm{NaHCO}_{3}, 0.4 \mathrm{NaH}_{2} \mathrm{PO}_{4}, 1.1 \mathrm{MgSO}_{4}, 11.1$ glucose and $0.04 \mathrm{Na}_{2}$ EDTA. This solution was

This article is protected by copyright. All rights reserved. 
equilibrated with $95 \% \mathrm{O}_{2}-5 \% \mathrm{CO}_{2}$ to give a $\mathrm{pH}$ of 7.4 , as described ${ }^{47}$. The mechanical activity of the heart was assessed by introducing into the left ventricle (LV) a balloon connected to a pressure transducer (AD Instruments MLT 0380, CO, USA) and filled with aqueous solution to achieve a left ventricular end-diastolic pressure (LVEDP) of approximately 5-10 $\mathrm{mmHg}$. LV contractility was evaluated using the developed-pressure (LVDP) and the maximal rate of pressure development $(+d P / d t)$. Relaxation was assessed by half relaxation time $\left(t_{1 / 2}\right)$, the time from peak developed pressure to $50 \%$ of decay of developed pressure ${ }^{47}$.

\section{Perfusion protocols}

Two experimental groups were performed: I/R 20/30 (hearts subjected to 20 min global ischemia followed by 30 min reperfusion) and I/R 30/60 (hearts subjected to $30 \mathrm{~min}$ global ischemia followed by $60 \mathrm{~min}$ reperfusion). At the end of reperfusion, hearts were freeze-clamped and stored at $-80^{\circ} \mathrm{C}$ for biochemical determinations. When drugs were used, they were perfused only during the reperfusion period [3 mM 4-phenylbutyrate (4-PBA) and $30 \mu \mathrm{M}$ tauroursodeoxycholic acid (TUDCA)]. A group of hearts perfused but not submitted to I/R protocol, was used as control.

\section{LDH determinations}

Lactate dehydrogenase (LDH) concentration was measured with a Boehring commercial kit (Boehring Mannheim, Mannheim, Germany) by spot sampling of the collected effluent at $10 \min ^{48}$.

\section{Measurement of lipid peroxidation}

Generation of ROS in the isolated rat heart was evaluated indirectly by measuring the formation of thiobarbituric acid reactants (TBARS), as an index of lipid peroxidation. Powered ventricular tissue from each heart was homogenized in 5 vol. of a buffer solution containing $140 \mathrm{mM} \mathrm{KCl}$ and $20 \mathrm{mM}$ $\mathrm{KH}_{2} \mathrm{PO}_{4}\left(\mathrm{pH} 7\right.$ at $\left.4^{\circ} \mathrm{C}\right)$ and then centrifuged at $1000 \times \mathrm{g}$ for $10 \mathrm{~min}$. The supernatant was used for the assay. The formation of TBARS was quantified according to Buege and Aust ${ }^{49}$.

\section{Electrophoresis and Western Blot}

Pulverized frozen ventricles were homogenized using a handheld homogenizer (Kinematica Polytron $1200 \mathrm{PT}$ E) in five volumes of the following: $20 \mathrm{mM}$ Tris-HCl, $5 \mathrm{mM}$ EDTA, $2.5 \mathrm{mM} \mathrm{EGTA}, 10 \mathrm{mM} \mathrm{NaCl}$, $300 \mathrm{mM}$ sucrose, 1\% IGEPAL, 0,1\% SDS, $25 \mathrm{mM} \mathrm{NaF}, 1 \mathrm{mM}$ PMSF, $1 \mathrm{mM}$ Benzamidine, $10 \mu \mathrm{g} / \mathrm{ml}$ Leupeptin, $1 \mu \mathrm{g} / \mathrm{ml}$ pepstatin, $1 \mu \mathrm{g} / \mathrm{ml}$ E64 [trans-Epoxysuccinyl-L-leucylamido(4-guanidino)butane]. The homogenate was sedimented at $13000 \mathrm{x}$ for $10 \mathrm{~min}$, and the supernatant was fractionated in small aliquots, frozen in liquid $\mathrm{N}_{2}$ and stored at $-80^{\circ} \mathrm{C}$. Protein concentration was measured using the Bradford method with bovine serum albumin (BSA) as the standard. $50 \mu \mathrm{g}$ of homogenate protein was electrophoresed per gel lane in 10-12\% acrylamide gels according to Laemmli. Proteins were transferred to PVDF membranes (Immobilion-P, Millipore). Blots were blocked with Tris-buffered

This article is protected by copyright. All rights reserved. 
saline (TBS) (50 mM Tris, $150 \mathrm{mM} \mathrm{NaCl}, \mathrm{pH}$ 7.4) with or without the addition of $0.1 \%$ Tween (TBST) and $5 \%$ skim milk or BSA, as indicated for each antibody, and then incubated with the following primary antibodies: phospho-JNK (1:1000 in TBST 5\% BSA), JNK (1:1000 in TBST 1\% milk), Santa Cruz Biotechnology, caspase-12 (1:1000 in TBST 1\% milk) Sigma-Aldrich, phospho-elF2 $\alpha$ (1:1000 in TBST $5 \%$ BSA) Cell Signaling Technology, elF2 $\alpha$ (1:1000 in TBST 1\% milk) Invitrogen, GAPDH (1:10000 in TBST 1\% milk) Millipore. The membranes were incubated with the appropriate horseradish peroxidase-conjugated secondary antibody (Santa Cruz Biotechnology, 1:10000 in 1\% milk TBST) and developed using an enhanced chemiluminescence reagent (Millipore). The signals emitted for chemiluminescence were detected using Chemidoc Imaging system (Bio-Rad) and analyzed with ImageJ software $(\mathrm{NIH})$. The results were normalized to corresponding densitometry signal of unphosphorylated protein or GAPDH.

\section{Real time qPCR}

RNA was extracted from hearts using TRIzol reagent (Life Technologies, Carlsbad, Calif). cDNA was generated by reverse transcriptase reaction using M-MLV RT (Promega, Madison, Wis). Real time quantitative PCR was performed on cDNA using the SYBR Select Master Mix (Life Technologies, Carlsbad, Calif) and iCycler iQ (Bio-Rad, Hercules, Calif). The following primers were used:

\begin{tabular}{|l|l|l|}
\hline Gene & Sense $\left(\mathbf{5}^{\prime}\right.$ to $\mathbf{3}^{\prime}$ ) & Antisense (5' to $\mathbf{3}^{\prime}$ ) \\
\hline $\begin{array}{l}\text { XBP1 (unspliced and spliced } \\
\text { forms) }\end{array}$ & AGAAACAGCAACCTGAGGGG & AGCAAGAAGATTCCATCAAGCAT \\
\hline CHOP & TACACCACCACACCTGAAAGC & GCTAGGGATGCAGGGTCAAG \\
\hline GRP78 & TGATTCCGAGGAACACTGTGG & GGTGATTGTCTTTTGTCAGGGG \\
\hline Spliced XBP1 & GAGTCCGCAGCAGGTGC & GGTCCAACTTGTCCAGAATGC \\
\hline GAPDH & GGGTGTGAACCACGAGAAAT & CCACAGTCTTCTGAGTGGCA \\
\hline
\end{tabular}

Relative abundance of RNA was calculated by the $\Delta \Delta \mathrm{Ct}$ method ${ }^{50}$. Primers were designed using Primer-Blast (NCBI, NIH). All primers were between $90 \%$ and $110 \%$ efficient, as assessed by standard curve, and all displayed only 1 dissociation peak.

\section{TUNEL technique}

The terminal deoxynucleotidyl transferase dUTP nick end labelling (TUNEL) technique was employed to study apoptosis, using the Tunel In situ Cell Death Detection Kit, TMR red (Roche, Indianapolis, IN, USA), as previously described ${ }^{48}$. Briefly, sections were deparaffinized, dehydrated and incubated with proteinase $\mathrm{K}$ for antigen retrieval. After washing with PBS containing 0.5\% Tween 20 (Merck, Schuchardt OHG, Hohenbrunn, Germany), slides were incubated with the reaction mixture containing modified nucleotides (TMR-dUTP) and the enzyme terminal deoxynucleotidyl transferase (TdT) that catalyses the template-independent polymerization of deoxyribonucleotides to the $3^{\prime}$-end of single and double-stranded DNA. After washing, nuclei were counterstained with $5 \mu \mathrm{g} \mathrm{ml}^{-1}$ of DAPI (6-diamidino-2-phenylindole; Invitrogen Life Technologies, Eugene, OR, USA) following the manufacturer's protocol. Cover slips were mounted on slides using the aqueous medium Reagent

This article is protected by copyright. All rights reserved. 
FluoroSave (Calbiochem, La Jolla, CA, USA) and then examined under confocal microscopy (FV1000, Olympus Co., Tokyo, Japan). The TUNEL reaction mixture replaced by the label solution of the kit was used as a negative control.

\section{Infarct size}

After the reperfusion period, hearts were perfused with $1 \%$ triphenyltetrazolium chloride (TTC) in

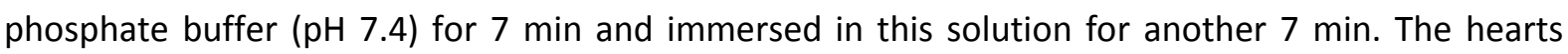
were frozen at $-20^{\circ} \mathrm{C}$ for $1 \mathrm{~h}$ and then cut into 6 transverse slices ( $2 \mathrm{~mm}$ thick) along the long axis of the LV, from apex to base. Infarct areas were enhanced by storage in $10 \%$ formaldehyde solution for $48 \mathrm{~h}$ before measurement. The quantification was performed on digital photographs by manually outlining the margins of the differentially colored areas: pale (infarcted) and red (viable tissue) using Image $J(\mathrm{NIH})$. Infarct weight was determined as follows: $(\mathrm{A} 1 \times \mathrm{W} 1)+(\mathrm{A} 2 \times \mathrm{W} 2)+(\mathrm{A} 3 \times \mathrm{W} 3)+(\mathrm{A} 4 \times$ $\mathrm{W} 4)+(\mathrm{A} 5 \times \mathrm{W} 5)+(\mathrm{A} 6 \times \mathrm{W} 6)$, where $A$ is the average infarct area of both sides of the slice and $\mathrm{W}$ the weight of the respective section. The weight of the area at risk (total ventricle area) was calculated in a similar fashion. Infarct size was expressed as the weight of infarct area/weight of ventricle area $x$ 100.

\section{Statistics}

Data are expressed as mean \pm SEM. Statistical significance was determined by Student's test for paired or unpaired observations as appropriate, and ANOVA when different groups were compared. The Newman-Keuls test was used to examine statistical differences observed with the ANOVA. A P value $<0.05$ was considered statistically significant.

\section{ACKNOWLEDGEMENTS}

We wish to thank Dr. Mariano Di Carlo for his initial collaboration with this project. B.R. is currently at Department of Pathology, Johns Hopkins University, Baltimore, MD, United States. We would like to thank Dr. Mariana Bollo for the generous gift of total elF2 $\alpha$ antibody. This work was supported by Agencia Nacional de Promoción Científica y Tecnológica, Argentina (PICT \# 2073 to L. Vittone and PICT \# 0856 to M. Said) and by Consejo de Investigaciones Científicas y Técnicas (CONICET), Argentina (PIP \# 0507 to C. Mundiña-Weilenmann).

\section{CONFLICT OF INTEREST}

No conflicts of interest, financial or otherwise, are declared by the authors.

This article is protected by copyright. All rights reserved. 


\section{REFERENCES}

1. Hausenloy DJ, Yellon DM. Myocardial ischemia-reperfusion injury: a neglected therapeutic target. J Clin Invest. 2013;123(1):92-100.

2. Groenendyk J, Sreenivasaiah PK, Kim DH, Agellon LB, Michalak M. Biology of endoplasmic reticulum stress in the heart. Circ Res. 2010;107(10):1185-1197.

3. Miyazaki $\mathrm{Y}$, Kaikita $\mathrm{K}$, Endo $\mathrm{M}$, et al. C/EBP homologous protein deficiency attenuates myocardial reperfusion injury by inhibiting myocardial apoptosis and inflammation. Arterioscler Thromb Vasc Biol. 2011;31(5):1124-1132.

4. Ogata $M$, Hino $S$, Saito $A$, et al. Autophagy is activated for cell survival after endoplasmic reticulum stress. Mol Cell Biol. 2006;26(24):9220-9231.

5. Sozen E, Karademir B, Ozer NK. Basic mechanisms in endoplasmic reticulum stress and relation to cardiovascular diseases. Free Radic Biol Med. 2015;78:30-41.

6. Wang $X, X u$ L, Gillette TG, Jiang $X$, Wang ZV. The unfolded protein response in ischemic heart disease. J Mol Cell Cardiol. 2018;117:19-25.

7. Doroudgar S, Thuerauf DJ, Marcinko MC, Belmont PJ, Glembotski CC. Ischemia activates the ATF6 branch of the endoplasmic reticulum stress response. I Biol Chem. 2009;284(43):29735-29745.

8. Martindale JJ, Fernandez R, Thuerauf $D$, et al. Endoplasmic reticulum stress gene induction and protection from ischemia/reperfusion injury in the hearts of transgenic mice with a tamoxifen-regulated form of ATF6. Circ Res. 2006;98(9):1186-1193.

9. Thuerauf DJ, Marcinko M, Gude N, Rubio M, Sussman MA, Glembotski CC. Activation of the unfolded protein response in infarcted mouse heart and hypoxic cultured cardiac myocytes. Circ Res. 2006;99(3):275-282.

10. Toko $\mathrm{H}$, Takahashi $\mathrm{H}$, Kayama $\mathrm{Y}$, et al. ATF6 is important under both pathological and physiological states in the heart. J Mol Cell Cardiol. 2010;49(1):113-120.

11. Belmont PJ, Chen WJ, San Pedro MN, et al. Roles for endoplasmic reticulum-associated degradation and the novel endoplasmic reticulum stress response gene Derlin-3 in the ischemic heart. Circ Res. 2010;106(2):307-316.

12. Vekich JA, Belmont PJ, Thuerauf DJ, Glembotski CC. Protein disulfide isomerase-associated 6 is an ATF6-inducible ER stress response protein that protects cardiac myocytes from ischemia/reperfusion-mediated cell death. J Mol Cell Cardiol. 2012;53(2):259-267.

13. Jin JK, Blackwood EA, Azizi K, et al. ATF6 Decreases Myocardial Ischemia/Reperfusion Damage and Links ER Stress and Oxidative Stress Signaling Pathways in the Heart. Circ Res. 2017;120(5):862-875.

14. Thuerauf DJ, Hoover $\mathrm{H}$, Meller J, et al. Sarco/endoplasmic reticulum calcium ATPase-2 expression is regulated by ATF6 during the endoplasmic reticulum stress response: intracellular signaling of calcium stress in a cardiac myocyte model system. J Biol Chem. 2001;276(51):48309-48317.

15. Wang ZV, Deng Y, Gao N, et al. Spliced X-box binding protein 1 couples the unfolded protein response to hexosamine biosynthetic pathway. Cell. 2014;156(6):1179-1192.

16. Smith MI, Deshmukh M. Endoplasmic reticulum stress-induced apoptosis requires bax for commitment and Apaf-1 for execution in primary neurons. Cell Death Differ. 2007;14(5):1011-1019.

17. Wang $\mathrm{Q}$, Zhang $\mathrm{H}$, Zhao B, Fei H. IL-1beta caused pancreatic beta-cells apoptosis is mediated in part by endoplasmic reticulum stress via the induction of endoplasmic reticulum $\mathrm{Ca2+}$ release through the c-Jun N-terminal kinase pathway. Mol Cell Biochem. 2009;324(1-2):183190.

18. Rutkowski DT, Kaufman RJ. That which does not kill me makes me stronger: adapting to chronic ER stress. Trends Biochem Sci. 2007;32(10):469-476.

This article is protected by copyright. All rights reserved. 
19. Jian L, Lu Y, Lu S, Lu C. Chemical Chaperone 4-Phenylbutyric Acid Reduces Cardiac Ischemia/Reperfusion Injury by Alleviating Endoplasmic Reticulum Stress and Oxidative Stress. Med Sci Monit. 2016;22:5218-5227.

20. Oveson BC, Iwase T, Hackett SF, et al. Constituents of bile, bilirubin and TUDCA, protect against oxidative stress-induced retinal degeneration. J Neurochem. 2011;116(1):144-153.

21. Bolli R, Marbán E. Molecular and cellular mechanisms of myocardial stunning. Physiol Rev. 1999;79(2):609-634.

22. Minamino T, Komuro I, Kitakaze M. Endoplasmic reticulum stress as a therapeutic target in cardiovascular disease. Circ Res. 2010;107(9):1071-1082.

23. Fujita E, Kouroku Y, Jimbo A, Isoai A, Maruyama K, Momoi T. Caspase-12 processing and fragment translocation into nuclei of tunicamycin-treated cells. Cell Death Differ. 2002;9(10):1108-1114.

24. Nakagawa T, Zhu H, Morishima N, et al. Caspase-12 mediates endoplasmic-reticulum-specific apoptosis and cytotoxicity by amyloid-beta. Nature. 2000;403(6765):98-103.

25. Obeng EA, Boise LH. Caspase-12 and caspase-4 are not required for caspase-dependent endoplasmic reticulum stress-induced apoptosis. J Biol Chem. 2005;280(33):29578-29587.

26. Saleh M, Vaillancourt JP, Graham RK, et al. Differential modulation of endotoxin responsiveness by human caspase-12 polymorphisms. Nature. 2004;429(6987):75-79.

27. Saleh M, Mathison JC, Wolinski MK, et al. Enhanced bacterial clearance and sepsis resistance in caspase-12-deficient mice. Nature. 2006;440(7087):1064-1068.

28. Sharma R, Gow A. Minimal role for caspase 12 in the unfolded protein response in oligodendrocytes in vivo. J Neurochem. 2007;101(4):889-897.

29. Roy S, Sharom JR, Houde C, et al. Confinement of caspase-12 proteolytic activity to autoprocessing. Proc Natl Acad Sci U S A. 2008;105(11):4133-4138.

30. Brown M, Strudwick N, Suwara M, et al. An initial phase of JNK activation inhibits cell death early in the endoplasmic reticulum stress response. J Cell Sci. 2016;129(12):2317-2328.

31. Cheng $\mathrm{X}$, Liu $\mathrm{H}$, Jiang $\mathrm{CC}$, et al. Connecting endoplasmic reticulum stress to autophagy through IRE1/JNK/beclin-1 in breast cancer cells. Int J Mol Med. 2014;34(3):772-781.

32. Raciti M, Lotti LV, Valia S, Pulcinelli FM, Di Renzo L. JNK2 is activated during ER stress and promotes cell survival. Cell Death Dis. 2012;3:e429.

33. Ventura JJ, Hübner A, Zhang C, Flavell RA, Shokat KM, Davis RJ. Chemical genetic analysis of the time course of signal transduction by JNK. Mol Cell. 2006;21(5):701-710.

34. Samali A, Fitzgerald U, Deegan S, Gupta S. Methods for monitoring endoplasmic reticulum stress and the unfolded protein response. Int J Cell Biol. 2010;2010:830307.

35. Sarvani C, Sireesh D, Ramkumar KM. Unraveling the role of ER stress inhibitors in the context of metabolic diseases. Pharmacol Res. 2017;119:412-421.

36. Luo T, Kim JK, Chen B, Abdel-Latif A, Kitakaze M, Yan L. Attenuation of ER stress prevents post-infarction-induced cardiac rupture and remodeling by modulating both cardiac apoptosis and fibrosis. Chem Biol Interact. 2015;225:90-98.

37. Wang $\mathrm{G}$, Huang $\mathrm{H}$, Zheng $\mathrm{H}$, et al. Zn2+ and mPTP Mediate Endoplasmic Reticulum Stress Inhibition-Induced Cardioprotection Against Myocardial Ischemia/Reperfusion Injury. Biol Trace Elem Res. 2016;174(1):189-197.

38. Wang ZH, Liu JL, Wu L, Yu Z, Yang HT. Concentration-dependent wrestling between detrimental and protective effects of $\mathrm{H} 2 \mathrm{O} 2$ during myocardial ischemia/reperfusion. Cell Death Dis. 2014;5:e1297.

39. $\mathrm{Yu} Y$, Sun $\mathrm{G}$, Luo $\mathrm{Y}$, et al. Cardioprotective effects of Notoginsenoside R1 against ischemia/reperfusion injuries by regulating oxidative stress- and endoplasmic reticulum stress- related signaling pathways. Sci Rep. 2016;6:21730.

40. Valverde CA, Kornyeyev D, Ferreiro M, Petrosky AD, Mattiazzi A, Escobar AL. Transient Ca2+ depletion of the sarcoplasmic reticulum at the onset of reperfusion. Cardiovasc Res. 2010;85(4):671-680.

This article is protected by copyright. All rights reserved. 
41. Hammadi M, Oulidi A, Gackière F, et al. Modulation of ER stress and apoptosis by endoplasmic reticulum calcium leak via translocon during unfolded protein response: involvement of GRP78. FASEB J. 2013;27(4):1600-1609.

42. Paredes RM, Bollo M, Holstein D, Lechleiter JD. Luminal Ca2+ depletion during the unfolded protein response in Xenopus oocytes: cause and consequence. Cell Calcium. 2013;53(4):286296.

43. Schäuble $N$, Lang $S$, Jung $M$, et al. BiP-mediated closing of the Sec61 channel limits Ca2+ leakage from the ER. EMBO J. 2012;31(15):3282-3296.

44. Carreras-Sureda A, Pihán P, Hetz C. Calcium signaling at the endoplasmic reticulum: finetuning stress responses. Cell Calcium. 2018;70:24-31.

45. Alder NN, Shen Y, Brodsky JL, Hendershot LM, Johnson AE. The molecular mechanisms underlying BiP-mediated gating of the Sec61 translocon of the endoplasmic reticulum. I Cell Biol. 2005;168(3):389-399.

46. Pomblum VJ, Korbmacher B, Cleveland S, Sunderdiek U, Klocke RC, Schipke JD. Cardiac stunning in the clinic: the full picture. Interact Cardiovasc Thorac Surg. 2010;10(1):86-91.

47. Vittone L, Mundiña-Weilenmann C, Said M, Ferrero P, Mattiazzi A. Time course and mechanisms of phosphorylation of phospholamban residues in ischemia-reperfused rat hearts. Dissociation of phospholamban phosphorylation pathways. I Mol Cell Cardiol. 2002;34(1):39-50.

48. Salas MA, Valverde CA, Sánchez G, et al. The signalling pathway of CaMKII-mediated apoptosis and necrosis in the ischemia/reperfusion injury. I Mol Cell Cardiol. 2010;48(6):1298-1306.

49. Buege JA, Aust SD. Microsomal lipid peroxidation. Methods Enzymol. 1978;52:302-310.

50. Pfaffl MW. A new mathematical model for relative quantification in real-time RT-PCR. Nucleic Acids Res. 2001;29(9):e45.

This article is protected by copyright. All rights reserved. 


\section{FIGURE LEGENDS}

Figure 1. mRNA expression of ER stress markers in the stunned heart. Total results of mRNA expression levels of sXBP1, total-XBP1, GRP78 and CHOP in non-ischemic hearts (Control) and hearts subjected to I/R 20/30 or I/R 30/60. Data represent means \pm SE of 7-14 hearts/group. ${ }^{*} \mathrm{p}<0.05$ respect to Control. \# $p<0.05$ vs. I/R 30/60.

Figure 2. Activation of proteins of the initial phase of ER stress response in the stunned heart. (A) Representative Western blots and (B) quantitative results of the phosphorylation of elF2 $\alpha$ and JNK and the cleavage of caspase 12 in non-ischemic hearts (Control, Ctrl) and hearts subjected to I/R $20 / 30$ or I/R 30/60. Data represent means \pm SE of 5-12 hearts/group. ${ }^{*} p<0.05$ respect to Control. \# p $<0.05$ vs. I/R $30 / 60$.

Figure 3. Assessment of cell apoptosis in hearts subjected to I/R. (A) Representative images of TUNEL (red dots) to denote the apoptotic cells, DAPI (blue dots) to mark the nuclei and the merging of both in a bright field image obtained from left ventricular sections of non-ischemic hearts (Ctrl) and hearts at the end of $\mathrm{I} / \mathrm{R}$ protocols. Yellow arrows highlight TUNEL-positive nuclei. (B) Quantitative analysis of TUNEL-positive nuclei. Data represent means \pm SE of 4-8 hearts/group.

Figure 4. Assessment of permanent cardiac damage in hearts subjected to I/R. (A) Representative images of TTC-stained rat heart sections obtained from non-ischemic hearts (Control, Ctrl) and hearts subjected to $\mathrm{I} / \mathrm{R} 20 / 30$ or $\mathrm{I} / \mathrm{R} 30 / 60$ and total results of the infarct size expressed as percentage of total ventricle area. (B) Total results of lactate dehydrogenase (LDH) release measured in Pre-Ischemia (Pre-Isch) and after $10 \mathrm{~min}$ of reperfusion in I/R protocols. Data represent means \pm SE of 4-10 hearts/group. ${ }^{*} p<0.05$ respect to Control. \# $p<0.05$ vs. I/R 30/60.

Figure 5. Effects of TUDCA on mRNA expression of ER stress markers and the pots-ischemic contractile recovery in the stunned heart. (A) Total results of mRNA expression levels of sXBP1 and GRP78. Data represent means \pm SE of $4-10$ hearts/group. ${ }^{*} p<0.05$ respect to Control. $\# p<0.05$ vs. I/R 20/30. (B-D) Time course of maximal rate of left ventricular pressure development (+dP/dt), half relaxation time $\left(t_{1 / 2}\right)$ and left ventricular end diastolic pressure (LVEDP) of hearts subjected to $\mathrm{I} / \mathrm{R}$ in the absence (I/R 20/30) or the presence of $30 \mu \mathrm{M}$ TUDCA (I/R 20/30 + TUDCA) applied at the beginning of reperfusion. Control: non-ischemic hearts. Data represent means \pm SE of 3-10 hearts/group. ${ }^{*} p<0.05$ respect to I/R 20/30.

Figure 6. Effects of 4-PBA on the post-ischemic contractile recovery of the stunned heart. (A) Total results of mRNA expression levels of SXBP1 and GRP78. Values are mean \pm SE of $4-9$ hearts. ${ }^{*} p<0.05$ respect to Control. \# p<0.05 vs. I/R 20/30. (B-D) Time course of maximal rate of left ventricular pressure development $(+d P / d t)$, half relaxation time $\left(t_{1 / 2}\right)$ and left ventricular end diastolic pressure (LVEDP) of hearts subjected to $I / R$ in the absence (I/R 20/30) or the presence of $3 \mathrm{mM} 4-P B A(I / R$ $20 / 30+4-P B A)$ applied upon reperfusion. Values are mean \pm SE of 5-10 hearts. ${ }^{*} p<0.05$ respect to I/R 20/30. (E) Quantification of the thiobarbituric acid reactive substances (TBARS) in Control (nonischemic hearts) and I/R 20/30 in the absence or the presence of 4-PBA or TUDCA. Values are expressed as means \pm SE of 3-9 hearts. ${ }^{*} p<0.05$ respect to Control. \# $p<0.05$ vs. I/R 20/30.

This article is protected by copyright. All rights reserved. 


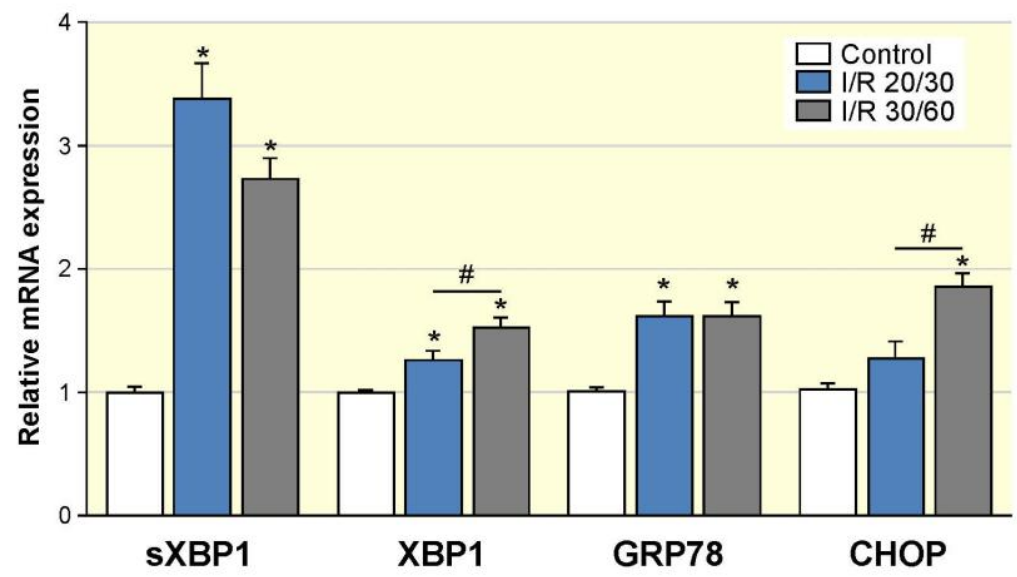

This article is protected by copyright. All rights reserved. 


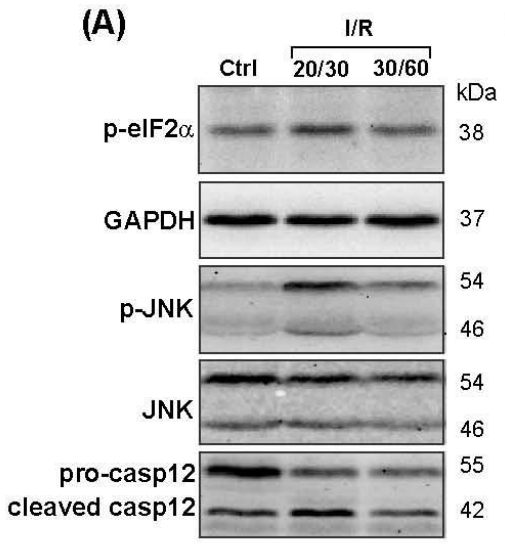

(B)

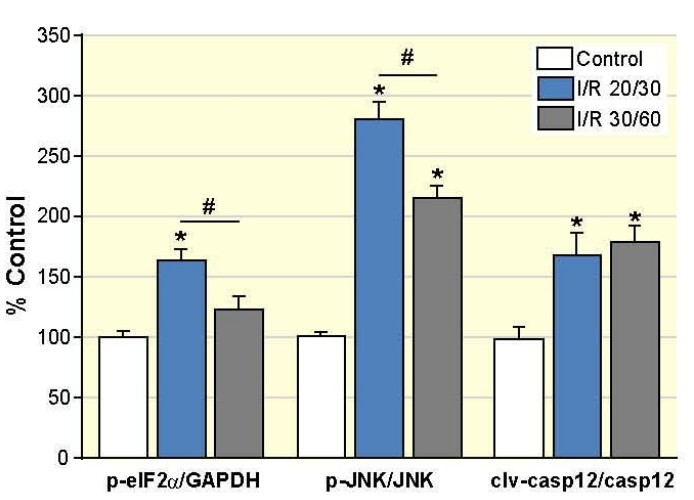

This article is protected by copyright. All rights reserved. 


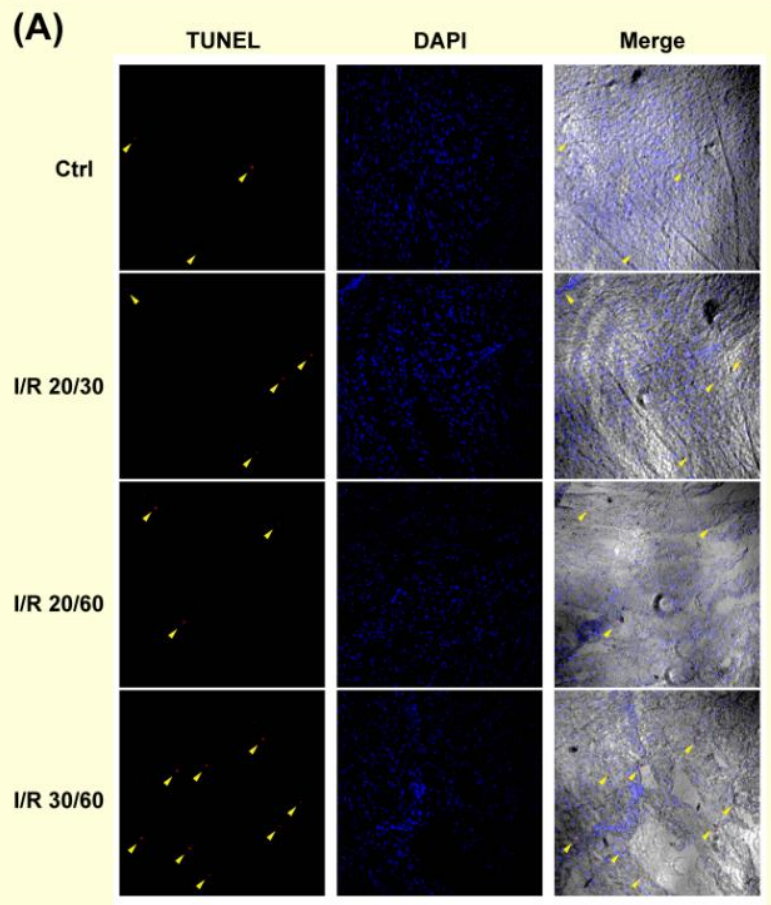

(B)

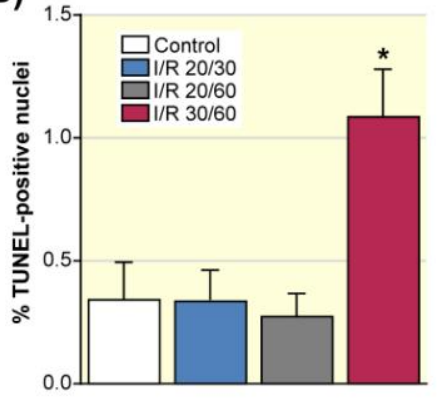



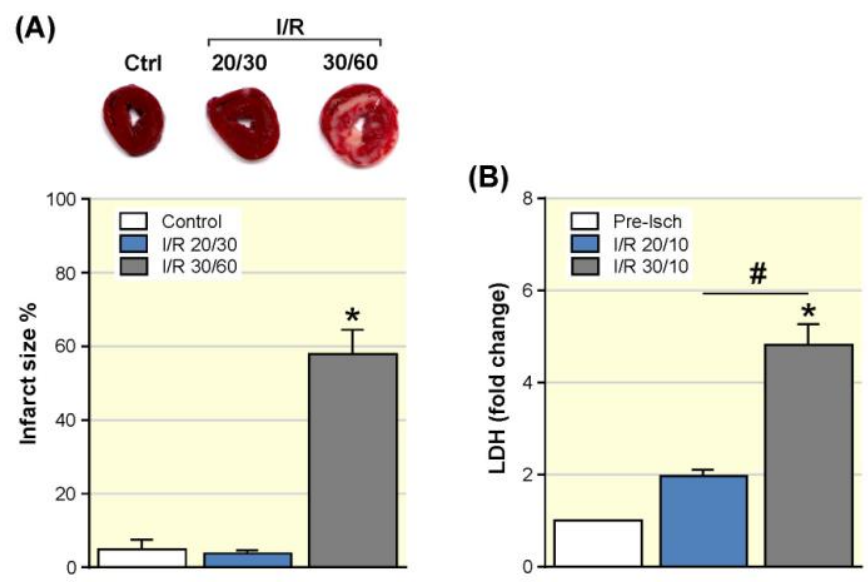

This article is protected by copyright. All rights reserved. 
(A)

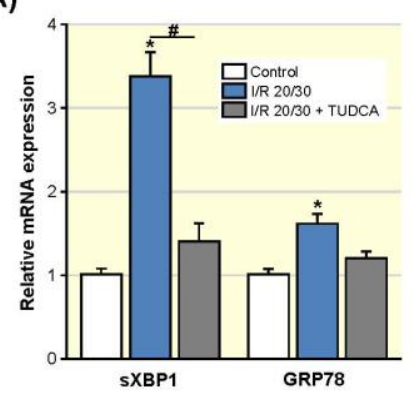

(C)

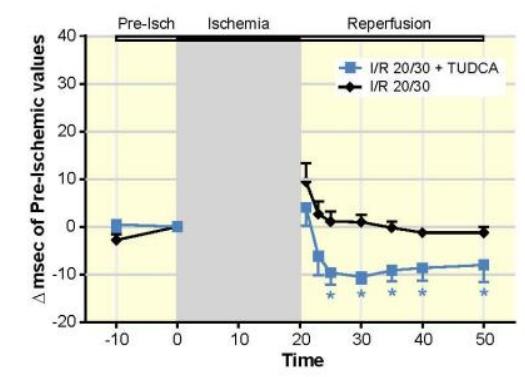

(B) $\quad+$ dP/dt

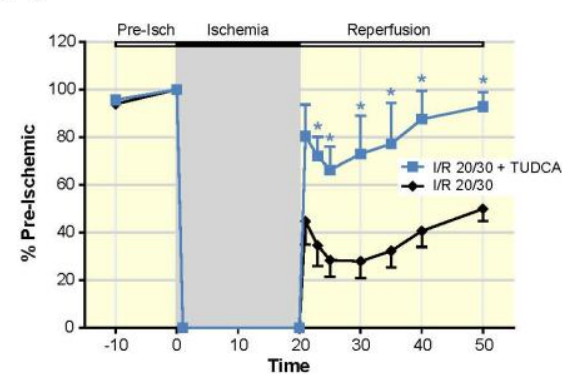

(D)

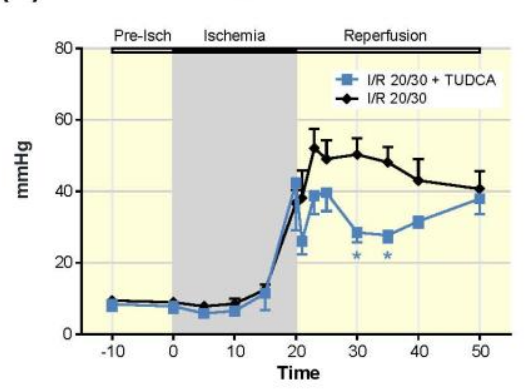

This article is protected by copyright. All rights reserved. 
(A)

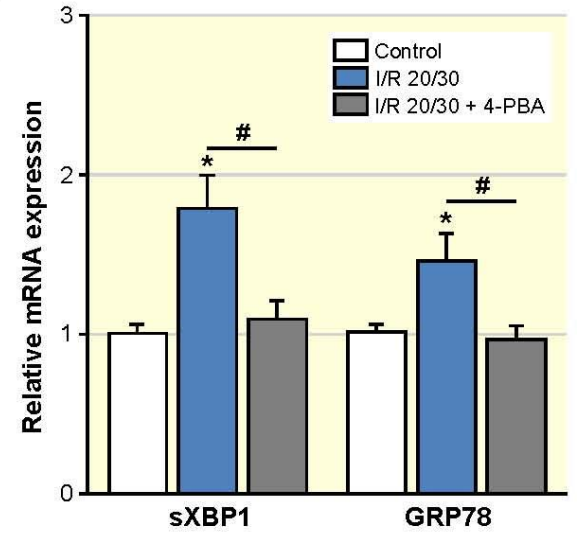

(C)

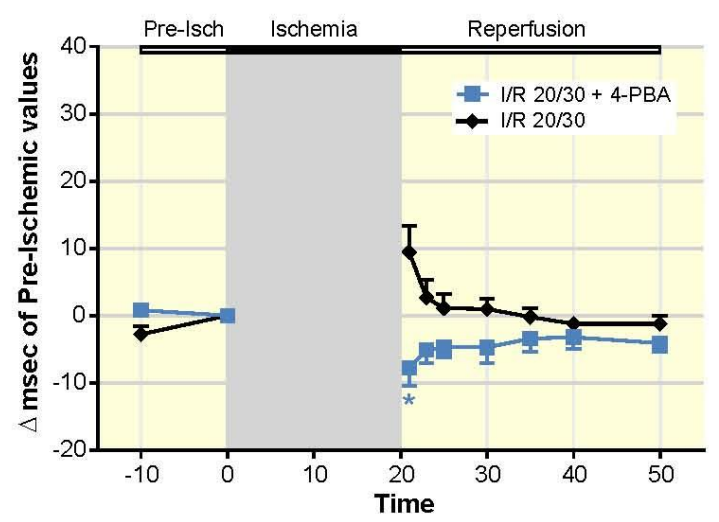

(E)

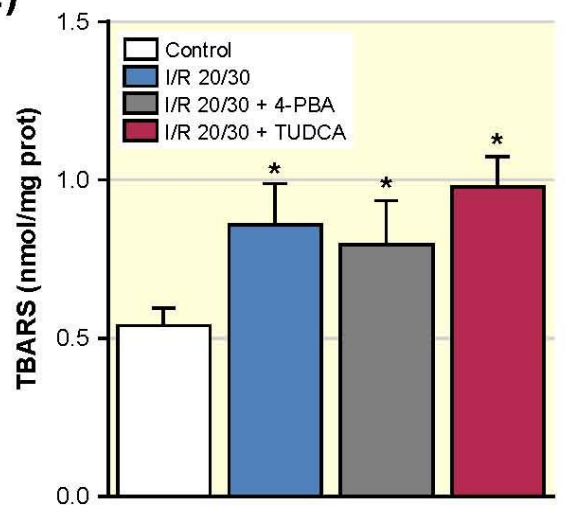

(B) $+d P / d t$

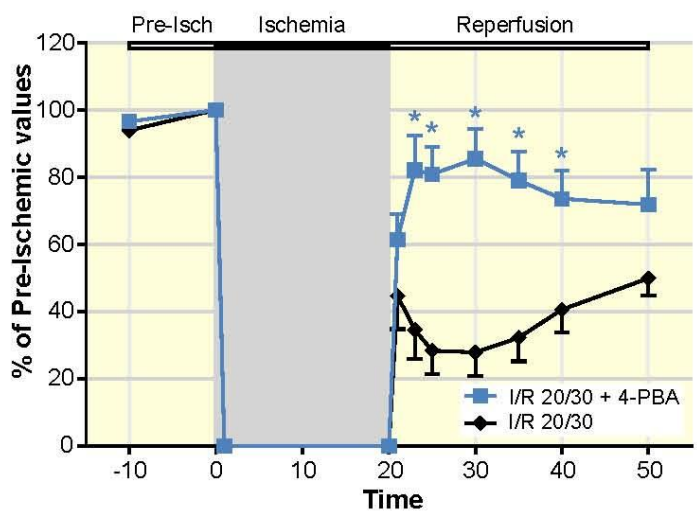

(D)

LVEDP

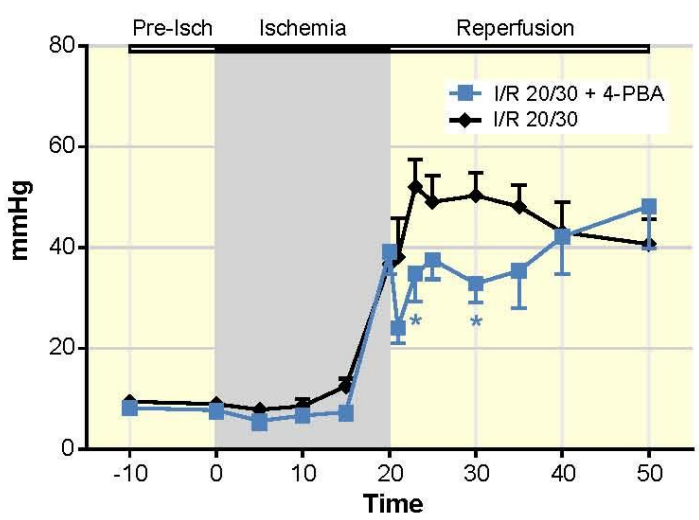

